\title{
A Partial Backlogging Two-Warehouse Inventory Models for Decaying Items with Inflation
}

\author{
${ }^{1}$ Dr. Ajay Singh Yadav, ${ }^{2}$ Ms. Anupam Swami \\ ${ }^{1}$ Assistant Professor, Department of Mathematics, SRM University NCR Campus, Ghaziabad, U.P \\ ${ }^{2}$ Assistant Professor, Department of Mathematics, Govt. Degree College, Sambhal, U.P
}

\begin{abstract}
An order level inventory model for decaying items with inventory level dependent demand rate. We have considered two cases: first is, model started with no shortages and second is model started from shortages. We have also taken the concept of inflation in this study. Finally, a numerical example for illustration is provided with sensitivity analysis.
\end{abstract}

\section{Introduction:}

The important problem associated with the inventory management is to decide where to stock the goods. This problem does not seem to have attracted much attention of researchers in this field. In the existing literature, it is found that classical inventory models generally deal with a single storage facility. The basic assumption in these models is that the management owned storage with unlimited capacity. In the field of inventory management, this is not always true. When an attracted price discount for bulk purchase is available or the cost of procuring goods is higher than the other inventory related costs, management then decides to purchase (or produce) a huge quantity of items at that time. All these items cannot be stored in the existing storage, i.e. in the owned warehouse (OW) with limited capacity. From economical point of view, they usually choose to rent other warehouses than rebuild a new warehouse. Thus, the excess quantities are stored in a rented warehouse (RW). This RW may be located near the OW or a little away from it. The inventory costs (including holding cost and deterioration cost) in RW are usually higher than those in OW due to additional cost of maintenance, material handling, etc. For example, the RW like "Central Warehousing Facility" generally provides better preserving facility than the OW resulting in a lower deterioration rate for the goods. To reduce the inventory costs, it will be economical to consume the goods of RW at the earliest. Consequently, the items are stored first in OW and only excess stock is stored in the RW. Further, the items of RW are transferred to OW in a continuous release pattern to meet the demand until the stock level in the RW is emptied and then the items of the OW are released.

Sarma (1983) proposed a two warehouse inventory model by assuming the cost of transporting K-unit from RW to OW as constant and called it as K-release rule (KRR). The rate of replenishment was assumed as infinite. Murdeshwar and Sathe (1985) formulated some aspects of lot size models with two level of storage and derived complete solution for optimum lot size under finite production rates. The authors assumed while deriving the K-release rule that $\mathrm{K}$ units were transferred $\mathrm{n}$-times from $\mathrm{OW}$ to $\mathrm{RW}$ during production stage with constant transportation cost. Sarma (1987) developed a deterministic inventory model for a single deteriorating item which was stored in two different warehouses of non deteriorating product. The preserving facilities were better in rented warehouse than own warehouse resulting in a lower rate of deterioration. Goswami and Chaudhuri (1992) developed an economic order quantity model for items with two levels of storage for a linear trend in demand. An inventory model for deteriorating items with two warehouses was formulated by Pakkala and Achary (1992). Pakkala and Achary (1994) proposed an inventory model for deteriorating products when two separate warehouses were used. A deterministic order level inventory model for deteriorating items with two storage facilities was discussed by Benkherouf (1997). Bhunia and Maiti (1998) developed a deterministic inventory model with two warehouses for deteriorating items taking linearly increasing demand with time, shortages were allowed and excess demand was backlogged as well. Yang (2004) developed the two-warehouse inventory models for deteriorating items with constant demand rate under inflation. An inventory model with two warehouses and stock-dependent demand rate was proposed by Zhou and Yang (2005). Shortages were not allowed in the model and the transportation cost for transferring items from RW to OW was taken to be dependent on the transported amount. Two-warehouse inventory models with LIFO and FIFO dispatching policies were developed by Lee (2006). Hsieh et al. (2008) suggested a deterministic inventory model for deteriorating items with two warehouses by minimizing the net present value of the total cost.

This chapter deals the inventory model for deteriorating items with stock-dependent demand rate, time-dependent holding cost and shortages under inflation, for two warehouses system in which each cycle begins with shortages and ends without shortages is considered. Then compare the two warehouses inventory models, one proposed and other the traditional one, based on the minimum cost approach we show that there 
exist a unique optimal solution to minimize the total relevant cost function. This study shows that when the inflation rate is greater than zero then our proposed model is less expensive than the traditional model. Finally, a numerical example for illustration is provided with sensitivity analysis.

\section{Assumptions and Notations:}

The following assumptions are used in this study:

1. Lead-time is zero and the initial inventory level is zero.

2. Deterioration is considered only after the inventory is stored in the warehouse.

3. There is no repair or replacement of the deteriorated inventory units.

4. The OW has a fixed capacity of w units and the RW has unlimited capacity.

5. Due to different stocking atmosphere, inventory cost (including carrying cost and deterioration cost) in RW are higher than those in OW.

6. Shortages are allowed and partially backlogged. The fraction of the shortages backordered is a differentiable and decreasing function of time $t$, denoted by $\delta(t)$ Where $t$ is the waiting time up to the next replenishment, with $0 \leq \delta(\mathrm{t}) \leq 1$ and $\delta(0)=1$. Note that if $\delta(\mathrm{t})=1$ (or 0$)$ for all $\mathrm{t}$, then shortages are completel backlogged (or lost).

7. When shortages are lost, the cost of lost sale is the sum of the revenue lost and the cost of lost goodwill. Hence the cost of lost sales here is grater than the unit purchase cost.

The notations used in this model are shown as follows:

$\mathrm{f}(\mathrm{t})=\mathrm{x}+\mathrm{y} \quad \mathrm{I}(\mathrm{t})$ demand rate.

w fixed capacity level of OW.

$\alpha \quad$ deterioration rate of inventory items in OW with $0<\alpha<1$.

$\beta \quad$ deterioration rate of inventory items in RW with $0<\beta<1$. $\beta>\alpha$

$\mathrm{r} \quad$ inflation rate.

$\mathrm{t}_{\mathrm{r}} \quad$ the time at which the inventory level reaches zero in $\mathrm{OW}$.

$\mathrm{t}_{\mathrm{o}} \quad$ the time at which the inventory level reaches zero in RW.

$\mathrm{t}_{\mathrm{s}} \quad$ the time at which the shortage level reaches the lowest point in the replenishment cycle.

$\mathrm{I}_{\mathrm{o}}(\mathrm{t}) \quad$ the inventory level in OW at time $\mathrm{t}$.

$\mathrm{I}_{\mathrm{r}}(\mathrm{t}) \quad$ the inventory level in RW at time $\mathrm{t}$.

$\mathrm{B}(\mathrm{t}) \quad$ the backlogged level at time $\mathrm{t}$.

$\delta(t) \quad$ the backlogging rate which is a decreasing function of the waiting time.

$\mathrm{C}_{\mathrm{o}} \quad$ the replenishment cost per order.

$\mathrm{C}_{\mathrm{b}}$ the backlogging cost per unit per unit time, if the shortages is backlogged.

$\mathrm{C}_{\mathrm{h} 1} \quad$ holding cost per unit per unit time in OW.

$\mathrm{C}_{\mathrm{h} 2} \quad$ holding cost per unit per unit time in RW.

$\mathrm{C}_{\mathrm{s}} \quad$ shortage cost per unit per unit time.

$\mathrm{C}_{1} \quad$ the unit opportunity cost due to lost sale, if the shortage is lost.

Note that if the objective is minimizing the total cost, then $C_{1}=p+C_{g}>C_{p}$, where $p$ is the cost of lost revenue and $\mathrm{C}_{\mathrm{g}}$ is the cost of lost goodwill, if the shortage is lost. Otherwise, if it is maximizing the total profit, then $\mathrm{C}_{1}=$ $\mathrm{C}_{\mathrm{g}}$.

$\mathrm{TC}_{\mathrm{i}} \quad$ the present value of the total relevant cost per unit time for model $\mathrm{i}, \mathrm{i}=1,2$

\section{Mathematical Model And Solution:}

There are two possible shortage models under the assumptions described above. In which, the traditional model (i.e., Model I) start with an instant order and ends with shortages, consequently Model II begins with shortages and ends without shortages. Discussion of both models with taken assumptions have been done as follows

\subsection{The mathematical formulation of the model starting with no Shortage:}

The inventory level, $\mathrm{I}(\mathrm{t}), 0 \leq \mathrm{t} \leq \mathrm{t}_{\mathrm{S}}$ satisfies the following differential equations with the corresponding boundary conditions :

$$
\begin{aligned}
& \frac{\mathrm{dI}_{\mathrm{r}}(\mathrm{t})}{\mathrm{dt}}+\beta \mathrm{I}_{\mathrm{r}}(\mathrm{t})=-\left(\mathrm{x}+\mathrm{y} \mathrm{I}_{\mathrm{r}}(\mathrm{t})\right), \quad \mathrm{O} \leq \mathrm{t} \leq \mathrm{t}_{\mathrm{r}}, \quad \mathrm{I}_{\mathrm{r}}\left(\mathrm{t}_{\mathrm{r}}\right)=\mathrm{O} . \\
& \frac{\mathrm{dI}_{\mathrm{O}}(\mathrm{t})}{\mathrm{dt}}=-\alpha \mathrm{I}_{\mathrm{O}}(\mathrm{t}), \quad 0 \leq \mathrm{t} \leq \mathrm{t}_{\mathrm{r}}, \quad \mathrm{I}_{\mathrm{O}}(\mathrm{O})=\mathrm{w} . \\
& \frac{\mathrm{dI_{O }}(\mathrm{t})}{\mathrm{dt}}+\alpha \mathrm{I}_{\mathrm{O}}(\mathrm{t})=-\left(\mathrm{x}+\mathrm{yI_{O }}(\mathrm{t})\right), \quad \mathrm{t}_{\mathrm{r}} \leq \mathrm{t} \leq \mathrm{t}_{\mathrm{O}}, \quad \mathrm{I}_{\mathrm{O}}\left(\mathrm{t}_{\mathrm{O}}\right)=\mathrm{O} .
\end{aligned}
$$


$\frac{\mathrm{dB}(\mathrm{t})}{\mathrm{dt}}=\delta\left(\mathrm{t}_{\mathrm{s}}-\mathrm{t}\right) \mathrm{x}, \quad \mathrm{t}_{\mathrm{O}} \leq \mathrm{t} \leq \mathrm{t}_{\mathrm{s}}, \quad \mathrm{B}\left(\mathrm{t}_{\mathrm{O}}\right)=0$

Inventory level

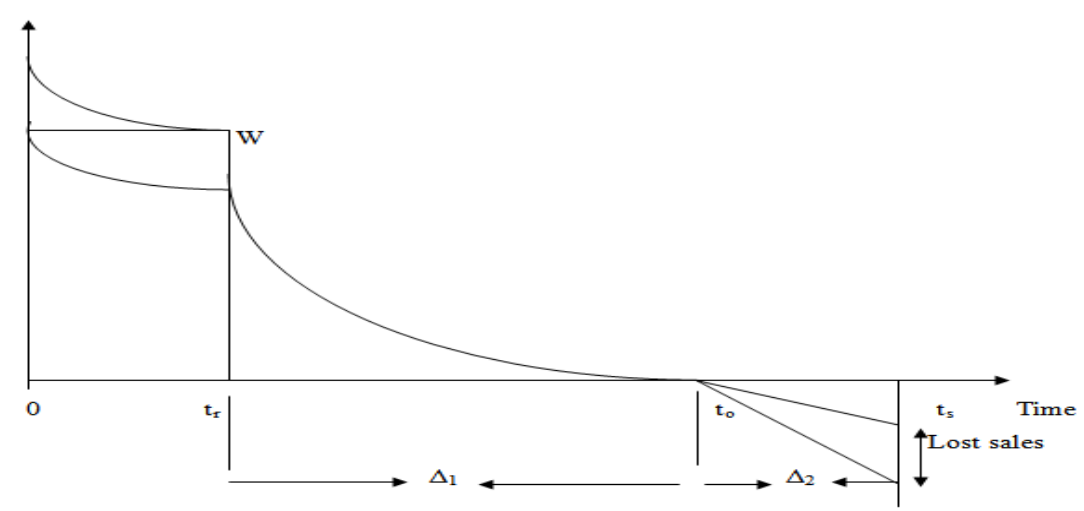

Fig. 1 Graphical representation of a two-warehouse inventory system for Model 1

Solutions of above equations are respectively,

$\mathbf{I}_{\mathbf{r}}(\mathbf{t})=\frac{\mathrm{x}}{(\boldsymbol{\beta + y})}\left[\mathrm{e}^{-(\boldsymbol{\beta}+\mathrm{y})\left(\mathrm{t}-\mathbf{t}_{\mathbf{r}}\right)}-\mathbf{1}\right], 0 \leq \mathrm{t} \leq \mathbf{t}_{\mathbf{r}}$.

$\mathrm{I}_{\mathbf{O}}(\mathrm{t})=\mathrm{we}^{-\alpha \mathrm{t}} \quad, \mathrm{O} \leq \mathbf{t} \leq \mathbf{t}_{\mathbf{r}}$.

(4.6)

$$
\begin{aligned}
& I_{O}(t)=\frac{x}{\alpha+y}\left[e^{-(\alpha+y)\left(t-t_{0}\right)}-1\right], t_{r} \leq t \leq t_{O} . \\
& B(t)=\int_{t_{0}}^{t} \delta\left(t_{s}-u\right) x d u \quad t_{0} \leq t \leq t_{s} .
\end{aligned}
$$

The number of lost sales at time $t$ is

$\mathbf{L}(\mathbf{t})=\int_{\mathbf{t}_{\mathbf{o}}}^{\mathbf{t}}\left[1-\delta\left(\mathrm{t}_{\mathrm{s}}-\mathbf{u}\right)\right] \mathrm{xdu}, \quad \mathbf{t}_{\mathrm{o}} \leq \mathrm{t} \leq \mathrm{t}_{\mathrm{s}}$.

Using the continuity of $I_{0}(t)$ at $t=t_{r}$, from eq. (4.6) \& (4.7), we have

$$
\begin{aligned}
& I_{0}\left(t_{r}\right)=w e^{-\alpha t_{r}}=\frac{x}{\alpha+y}\left[e^{-(\alpha+y)\left(t_{r}-t_{0}\right)}-1\right] \\
& \Rightarrow w=\frac{x^{\alpha t_{r}}}{\alpha+y}\left[e^{-(\alpha+y)\left(t_{r}-t_{0}\right)}-1\right]
\end{aligned}
$$

$$
\Rightarrow \mathrm{t}_{\mathrm{O}}=\mathrm{t}_{\mathrm{r}}+\frac{1}{(\alpha+\mathrm{y})} \ln \left(1+\frac{(\alpha+\mathrm{y}) \mathrm{we}^{-\alpha \mathrm{t}_{\mathrm{r}}}}{\mathrm{x}}\right)
$$

Therefore $\mathbf{t}_{\mathbf{O}}$ is not a decision variable in model I. Thus, the cumulative inventories in RW during $\left(\mathbf{O}, \mathbf{t}_{\mathbf{r}}\right)$ and in OW during $\left(\mathrm{O}, \mathrm{t}_{\mathbf{O}}\right)$ are

$$
\begin{aligned}
& \int_{O}^{t^{t}} r_{r} I_{r}(t) d t=\int_{O}^{t} r \frac{x}{\beta+y}\left[e^{-(\beta+y)\left(t-t_{r}\right)}-1\right] d t \\
& =\frac{x}{(\beta+y)}\left[\frac{e^{(\beta+y) t_{r}}}{(\beta+y)}\left(1-e^{-(\beta+y) t_{r}}\right)-t_{\mathbf{r}}\right] \\
& \int_{O}^{t_{r}} I_{r}(t) d t=\frac{x}{\beta+y}\left[\frac{1}{\beta+y}\left(e^{(\beta+y) t_{r}}-1\right)-t_{r}\right] \\
& \int_{O}^{t_{O}} I_{O}(t) d t=\int_{O}^{t^{t}} r_{w e}-\alpha t d t+\int_{t_{r}}^{t_{O}} \frac{x}{\alpha+y}\left[e^{-(\alpha+y)\left(t-t_{0}\right)}-1\right] d t \\
& =\frac{w}{\alpha}\left(1-e^{-\alpha t_{r}}\right)+\frac{x}{(\alpha+y)}\left[\frac{e^{(\alpha+y) t_{0}}}{(\alpha+y)}\left(e^{-(\alpha+y) t_{r}}-e^{-(\alpha+y) t_{0}}\right)-\left(t_{\mathbf{o}}-t_{r}\right)\right]
\end{aligned}
$$




$$
\begin{aligned}
& =\frac{w}{\alpha}\left(1-e^{-\alpha t_{r}}\right)+\frac{x}{(\alpha+y)}\left[\frac{1}{(\alpha+y)}\left(e^{-(\alpha+y)\left(t_{r}-t_{0}\right)}-1\right)-\left(t_{0}-t_{r}\right)\right] \\
& =\frac{\mathrm{w}}{\alpha}-\frac{\mathrm{x}}{\alpha(\alpha+\mathrm{y})}\left(\mathrm{e}^{-(\alpha+\mathrm{y})\left(\mathrm{t}_{\mathrm{r}}-\mathrm{t}_{\mathbf{0}}\right)}-1\right)+ \\
& \frac{\mathrm{x}}{(\alpha+\mathrm{y})}\left[\frac{1}{(\alpha+\mathrm{y})}\left(\mathrm{e}^{-(\alpha+\mathrm{y})\left(\mathrm{t}_{\mathbf{r}}-\mathrm{t}_{\mathrm{o}}\right)}-1\right)-\left(\mathrm{t}_{\mathrm{o}}-\mathrm{t}_{\mathbf{r}}\right)\right] \\
& =\frac{w}{\alpha}-\frac{x}{\alpha+y}\left(\frac{1}{\alpha}-\frac{1}{\alpha+y}\right)\left(e^{-(\alpha+y)\left(t_{r}-t_{0}\right)}-1\right)-\frac{x}{\alpha+y}\left(t_{0}-t_{r}\right) \\
& =\frac{w}{\alpha}-\frac{x y}{\alpha(\alpha+y)^{2}}\left(e^{-(\alpha+y)\left(t_{r}-t_{0}\right)}-1\right)-\frac{x}{(\alpha+y)}\left(t_{0}-t_{r}\right)
\end{aligned}
$$

Respectively, and the present value of the inventory holding cost in RW and OW are

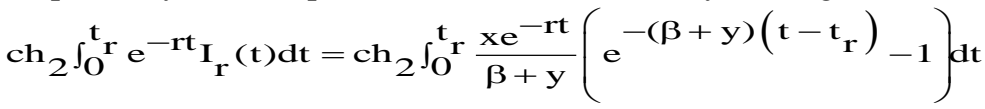

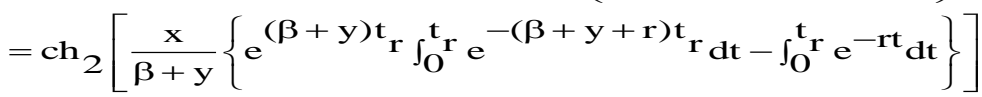

$$
\begin{aligned}
& =\frac{\mathbf{x c h}_{2}}{\beta+\mathbf{y}}\left[\frac{\mathrm{e}^{(\beta+y) \mathbf{t}_{\mathbf{r}}}}{(\beta+\mathbf{y}+\mathbf{r})}\left(1-\mathrm{e}^{-(\beta+\mathbf{y}+\mathbf{r}) \mathbf{t}_{\mathbf{r}}}\right)-\frac{\mathbf{1}}{\mathbf{r}}\left(1-\mathrm{e}^{-\mathbf{r t} \mathbf{r}}\right)\right]
\end{aligned}
$$

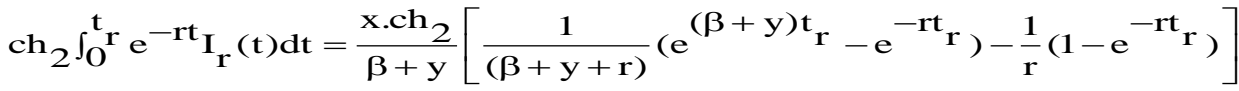

and

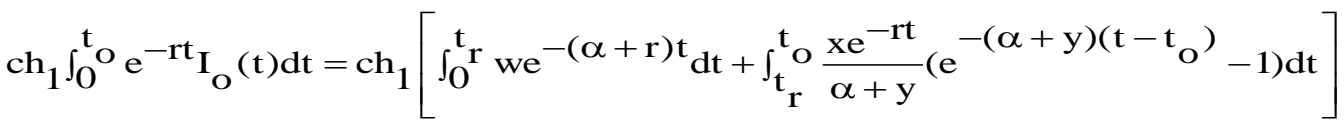

$$
\begin{aligned}
& =\operatorname{ch}_{1}\left[\int_{0}^{t_{r}} \frac{\mathrm{xe}^{\alpha t_{r}}}{\alpha+y}\left(e^{-(\alpha+y)\left(t_{r}-t_{0}\right)}-1\right) e^{-(\alpha+r) t} d t\right.
\end{aligned}
$$

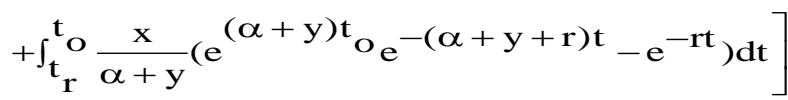

$$
\begin{aligned}
& =\operatorname{ch}_{1}\left[\frac{\mathrm{xe}^{\alpha \mathrm{t}_{\mathbf{r}}}}{\alpha+\mathrm{y}}\left(\mathrm{e}^{-(\alpha+\mathrm{y})\left(\mathrm{t}_{\mathbf{r}}-\mathrm{t}_{\mathbf{o}}\right)}-1\right)\left(\frac{1-\mathrm{e}^{-(\alpha+\mathrm{r}) \mathrm{t}_{\mathbf{r}}}}{\alpha+\mathrm{r}}\right)\right. \\
& \left.+\frac{x}{\alpha+y}\left\{e^{(\alpha+y) t_{o}}\left(\frac{e^{-(\alpha+y+r) t_{r}}-e^{-(\alpha+y+r) t_{o}}}{(\alpha+y+r)}\right)-\frac{1}{r}\left(e^{-r t_{r}}-e^{-r \mathbf{t}_{\mathbf{o}}}\right)\right\}\right] \\
& =\frac{x \cdot c h_{1}}{\alpha+y}\left[\frac{e^{\alpha t_{r}}}{\alpha+r}\left(e^{-(\alpha+y)\left(t_{r}-t_{0}\right)}-1\right)\left(1-e^{-(\alpha+r) t_{r}}\right)\right. \\
& \left.+\frac{\mathrm{e}^{(\alpha+y) t} \mathrm{o}}{(\alpha+\mathrm{y}+\mathrm{r})}\left(\mathrm{e}^{-(\alpha+\mathrm{y}+\mathrm{r}) \mathrm{t}} \mathrm{r}-\mathrm{e}^{-(\alpha+\mathrm{y}+\mathrm{r}) \mathrm{t}} \mathrm{o}\right)-\frac{1}{\mathrm{r}}\left(\mathrm{e}^{-\mathrm{rt}} \mathrm{r}-\mathrm{e}^{-\mathrm{rt}} \mathrm{o}\right)\right]
\end{aligned}
$$

Respectively and the present value of the backlogging cost and the opportunity cost due to lost sale are $c_{b} \int_{t_{0}}^{t} s^{-r t} \int_{t_{0}}^{t} \delta\left(t_{s}-u\right) x d u d t=\frac{c_{b}}{r} \int_{t}^{t} s\left(e^{-r t}-e^{-r t} s\right) \delta\left(t_{s}-t\right) x d t$

(4.16) And

$$
c_{1} e^{-r t_{S}} \int_{t_{o}}^{t}\left[1-\delta\left(t_{S}-t\right)\right] x d t
$$

In addition, the present value of the cost for the deteriorated items is

$$
c_{p}\left[\beta \int_{O}^{t} r^{r} e^{-r t} I_{r}(t) d t+\alpha \int_{O}^{t} e^{-r t} I_{o}(t) d t\right]
$$




$$
\begin{aligned}
& =c_{p}\left[\frac{\beta x}{\beta+y}\left\{\frac{1}{\beta+y+r}\left(e^{(\beta+y) t} r-e^{-r t} r\right)-\frac{1}{r}\left(1-e^{-r t} r\right)\right\}\right. \\
& +\frac{\alpha x}{\alpha+y}\left\{\frac{e^{\alpha t_{r}}}{\alpha+r}\left(e^{-(\alpha+y)\left(t_{r}-t_{0}\right)}-1\right)\left(1-e^{-(\alpha+r) t_{r}}\right)+\right. \\
& \left.\left.+\frac{\mathrm{e}^{(\alpha+y) t} \mathrm{o}}{\alpha+\mathrm{y}+\mathrm{r}}\left(\mathrm{e}^{-(\alpha+\mathrm{y}+\mathrm{r}) \mathrm{t}} \mathrm{r}-\mathrm{e}^{-(\alpha+\mathrm{y}+\mathrm{r}) \mathrm{t}} \mathrm{o}\right)-\frac{1}{\mathrm{r}}\left(\mathrm{e}^{-\mathrm{rt}} \mathrm{r}-\mathrm{e}^{-\mathrm{rt}} \mathrm{o}\right)\right\}\right]
\end{aligned}
$$

Consequently, the present value of the total cost per unit time is given by

$$
\begin{aligned}
& \mathrm{TC}_{1}=\frac{1}{\left(\mathrm{t}_{\mathrm{r}}+\Delta_{1}+\Delta_{2}\right)}\left[\mathrm{c}_{\mathrm{o}}+\frac{\left(\mathrm{ch}_{2}+\beta \mathrm{c}_{\mathrm{p}}\right) \mathrm{x}}{\beta+\mathrm{y}}\left\{\frac{\mathrm{e}^{(\beta+\mathrm{y}) \mathrm{t}_{\mathrm{r}}-\mathrm{e}^{-\mathrm{rt}_{\mathrm{r}}}}}{\beta+\mathrm{y}+\mathrm{r}}-\frac{1-\mathrm{e}^{-\mathrm{rt}} \mathrm{r}}{\mathrm{r}}\right\}\right. \\
& -\frac{\left(\mathrm{ch}_{1}+\alpha \mathrm{c}_{\mathrm{p}}\right) \times}{(\alpha+\mathrm{y})}\left\{\frac{\mathrm{e}^{-\mathrm{rt}} \mathrm{r}}{(\alpha+\mathrm{r})}\left(\mathrm{e}^{(\alpha+\mathrm{y}) \Delta_{1}}-1\right)-\frac{\mathrm{e}^{-\mathrm{rt}} \mathrm{o}}{(\alpha+\mathrm{y}+\mathrm{r})}\left(\mathrm{e}^{-(\alpha+\mathrm{y}+\mathrm{r}) \Delta_{1}}-1\right)\right. \\
& \left.+\frac{\mathrm{e}^{-\mathrm{rt}} \mathrm{o}}{\mathrm{r}}\left(\mathrm{e}^{\mathrm{r} \Delta_{1}}-1\right)\right\}+\frac{\left(\mathrm{ch}_{1}+\alpha \mathrm{c}_{\mathrm{p}}\right) \mathrm{w}}{(\alpha+\mathrm{r})} \\
& \left.+x e^{-r\left(t_{r}+\Delta_{1}+\Delta_{2}\right)} \int_{t_{0}}^{t_{j}} \frac{c_{b}}{r}\left\{\left(e^{r\left(t_{s}-t\right)}-1\right) \delta\left(t_{s}-t\right)+c_{1}\left(1-\delta\left(t_{s}-t\right)\right)\right\} d t\right]
\end{aligned}
$$

Where from fig.1, $\Delta_{1}=\mathrm{t}_{\mathrm{o}}-\mathrm{t}_{\mathrm{r}}, \Delta_{2}=\mathrm{t}_{\mathrm{s}}-\mathrm{t}_{\mathrm{o}}$ and $\mathrm{w}=\frac{\mathrm{xe}}{\alpha+\mathrm{y}}\left[\mathrm{e}^{-(\alpha+\mathrm{y})\left(\mathrm{t}_{\mathrm{r}}-\mathrm{t}_{\mathrm{o}}\right)}-1\right]$

\subsection{The mathematical formulation of the model starting with Shortages:}

In this section the inventory model starting with shortages is studied. The cycle now starts with shortages, which occur during the period $\left(0, t_{\mathrm{s}}^{\prime}\right)$. At time $\mathrm{t}_{\mathrm{s}}^{\prime}$ replenishment fills $\mathrm{OW}$ and RW. To distinguish easily, the notations $\mathbf{t}_{\mathbf{O}}, \mathbf{t}_{\mathbf{r}}, \mathbf{t}_{\mathrm{s}}$ are replaced by $\mathbf{t}_{\mathrm{r}}^{\prime}, \mathrm{t}_{\mathrm{s}}^{\prime}, \mathrm{t}_{\mathrm{o}}^{\prime}$ in model II. For Model II, the present value of ordering cost ${ }^{\text {is }} \mathrm{c}_{\mathbf{O}} \mathrm{e}^{-\mathbf{r t}_{\mathrm{s}}^{\prime}}$. The inventory level, $\mathrm{I}(\mathrm{t}), \mathrm{O} \leq \mathbf{t} \leq \mathrm{t}_{\mathrm{o}}^{\prime}$ satisfies the following differential equations:

$$
\begin{aligned}
& \frac{d I_{r}(t)}{d t}+\beta I_{r}(t)=-\left(x+y I_{r}(t)\right), t_{s}^{\prime} \leq t \leq t_{r}^{\prime}, I_{r}\left(t_{r}^{\prime}\right)=0 . \\
& \frac{d I_{0}(t)}{d t}+\alpha I_{0}(t)=0, \quad t_{s}^{\prime} \leq t \leq t_{r}^{\prime}, \quad I_{0}\left(t_{s}^{\prime}\right)=w . \\
& \frac{d I_{O}(t)}{d t}+\alpha I_{0}(t)=-\left(x+y I_{0}(t)\right), t_{r}^{\prime} \leq t \leq t_{0}^{\prime}, \quad I_{O}\left(t_{0}^{\prime}\right)=0 \\
& \frac{d B(t)}{d t}=\delta\left(t_{s}-t\right) x, \quad 0 \leq t \leq t_{s}^{\prime}, \quad B(0)=0 .
\end{aligned}
$$

Solutions of above equations are respectively,

$$
\begin{aligned}
& I_{r}(t)=\frac{x}{\beta+y}\left[e^{-(\beta+y)\left(t-t_{r}^{\prime}\right)}-1\right], \quad t_{s}^{\prime} \leq t \leq t_{r}^{\prime} . \\
& I_{0}(t)=w e^{\alpha\left(t_{s}^{\prime}-t\right)}, \quad t_{s}^{\prime} \leq t \leq t_{r}^{\prime} .
\end{aligned}
$$

$$
I_{0}(t)=\frac{x}{\alpha+y}\left[e^{-(\alpha+y)\left(t-t_{0}^{\prime}\right)}-1\right], \quad t_{r}^{\prime} \leq t \leq t_{0}^{\prime}
$$

$$
\mathrm{B}(\mathrm{t})=\int_{0}^{\mathrm{t}} \delta\left(\mathrm{t}_{\mathrm{s}}-\mathrm{u}\right) \mathrm{xdu}, \quad 0 \leq \mathrm{t} \leq \mathrm{t}_{\mathrm{s}}^{\prime} .
$$

The number of lost sales at time $t$ is 
$\mathrm{L}(\mathrm{t})=\int_{\mathrm{O}}^{\mathrm{t}}\left[1-\delta\left(\mathrm{t}_{\mathrm{s}}-\mathrm{u}\right)\right] \mathrm{xdu}, \quad 0 \leq \mathrm{t} \leq \mathrm{t}_{\mathrm{S}}^{\prime}$.

(4.27)

Inventory level

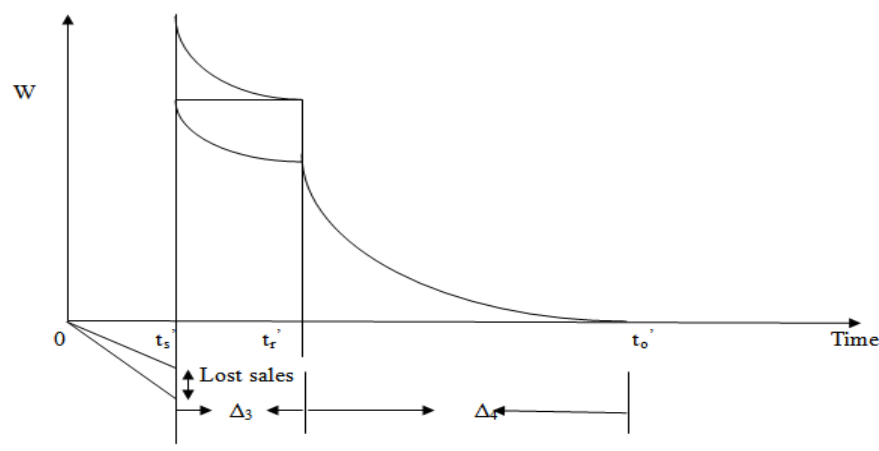

Fig. 2 Graphical representation of a two-warehouse inventory system for Model 2

Using the continuity of $\mathbf{I}_{\mathbf{O}}(\mathbf{t})$ at $\mathrm{t}=\mathbf{t}_{\mathbf{r}}^{\prime}$, from eq. (4.24) \& (4.25), we have

$$
\begin{aligned}
& I_{0}\left(t_{r}^{\prime}\right)=\mathrm{we}^{\alpha\left(\mathrm{t}_{\mathrm{s}}^{\prime}-\mathrm{t}_{\mathrm{r}}^{\prime}\right)}=\frac{\mathrm{x}}{\alpha+\mathrm{y}}\left[\mathrm{e}^{-(\alpha+\mathrm{y})\left(\mathrm{t}_{\mathrm{r}}^{\prime}-\mathrm{t}_{\mathrm{o}}^{\prime}\right)}-1\right] \\
& \Rightarrow \mathrm{w}=\frac{\mathrm{xe}}{-\alpha\left(\mathrm{t}_{\mathrm{s}}^{\prime}-\mathrm{t}_{\mathrm{r}}^{\prime}\right)}\left[\mathrm{e}^{-(\alpha+\mathrm{y})\left(\mathrm{t}_{\mathrm{r}}^{\prime}-\mathrm{t}_{\mathrm{o}}^{\prime}\right)_{-1]}}\right. \\
& \Rightarrow \mathrm{t}_{\mathrm{O}}^{\prime}=\mathrm{t}_{\mathrm{r}}^{\prime}+\frac{1}{\alpha+\mathrm{y}} \ln \left(1+\frac{(\alpha+\mathrm{y}) \mathrm{we} \mathrm{e}^{\alpha\left(\mathrm{t}_{\mathrm{s}}^{\prime}-\mathrm{t}_{\mathrm{r}}^{\prime}\right)}}{\mathrm{x}}\right)
\end{aligned}
$$

Therefore $\mathbf{t}_{\mathbf{o}}^{\prime}$ is not a decision variable in model II. Thus, the cumulative inventories in RW during $\left(\mathrm{t}_{\mathrm{s}}^{\prime}, \mathbf{t}_{\mathbf{r}}^{\prime}\right)$ and in OW during $\left(t_{s}^{\prime}, t_{0}^{\prime}\right)$ are

$$
\begin{aligned}
& \int_{t_{s}^{\prime}}^{t_{r}^{\prime}} I_{r}(t) d t=\int_{t_{s}^{\prime}}^{t_{r}^{\prime}} \frac{x}{\beta+y}\left\{e^{-(\beta+y)\left(t-t_{r}^{\prime}\right)}-1\right\} d t \\
& =\frac{x}{\beta+y}\left[\frac{e^{-(\beta+y)\left(t-t_{r}^{\prime}\right)}}{-(\beta+y)}-t\right]_{t_{s}^{\prime}}^{t_{r}^{\prime}} \\
& \Rightarrow \int_{t_{S}^{\prime}}^{t_{r}^{\prime}} I_{r}(t) d t=\frac{x}{\beta+y}\left[\frac{1}{\beta+y}\left(e^{-(\beta+y)\left(t_{s}^{\prime}-t_{r}^{\prime}\right)}-1\right)-\left(t_{r}^{\prime}-t_{s}^{\prime}\right)\right]
\end{aligned}
$$

And

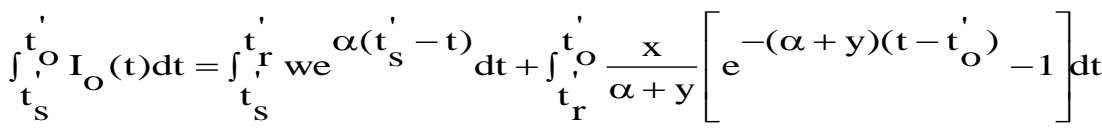

$=w e^{\alpha t^{\prime}} \int_{t_{s}}^{t_{1}^{\prime}} e^{-\alpha t} d t+\frac{x}{\alpha+y} \int_{t_{r}^{\prime}}^{t^{\prime}}\left[e^{(\alpha+y) t_{o}^{\prime}} e^{-(\alpha+y) t}-1\right] d t$

$=\frac{w e^{\alpha t_{s}^{\prime}}}{\alpha}\left(e^{-\alpha t_{s}^{\prime}}-e^{-\alpha t_{r}^{\prime}}\right)+\frac{x}{(\alpha+y)}\left[\frac{e^{(\alpha+y) t_{0}^{\prime}}}{(\alpha+y)}\left(e^{-(\alpha+y) t_{r}^{\prime}}-e^{-(\alpha+y) t_{0}^{\prime}}\right)-\left(t_{0}^{\prime}-t_{r}^{\prime}\right)\right]$

$=\frac{w}{\alpha}\left(1-\mathrm{e}^{-\alpha\left(\mathrm{t}_{\mathrm{r}}^{\prime}-\mathrm{t}_{\mathrm{s}}^{\prime}\right)}\right)+\frac{\mathrm{x}}{(\alpha+\mathrm{y})}\left[\frac{1}{(\alpha+\mathrm{y})}\left(\mathrm{e}^{-(\alpha+\mathrm{y})\left(\mathrm{t}_{\mathrm{r}}^{\prime}-\mathrm{t}_{\mathrm{O}}^{\prime}\right)}-1\right)-\left(\mathrm{t}_{\mathrm{o}}^{\prime}-\mathrm{t}_{\mathrm{r}}^{\prime}\right)\right]$

$=\frac{w}{\alpha}-\frac{x^{-\alpha\left(t_{s}^{\prime}-t_{r}^{\prime}\right)}}{\alpha(\alpha+y)}\left[e^{-(\alpha+y)\left(t_{r}^{\prime}-t_{o}^{\prime}\right)}-1\right] e^{-\alpha\left(t_{r}^{\prime}-t_{s}^{\prime}\right)}$ 


$$
\begin{aligned}
& +\frac{\mathrm{x}}{(\alpha+\mathrm{y})}\left[\frac{1}{(\alpha+\mathrm{y})}\left(\mathrm{e}^{-(\alpha+\mathrm{y})\left(\mathrm{t}_{\mathrm{r}}^{\prime}-\mathrm{t}_{\mathrm{o}}^{\prime}\right)}-1\right)-\left(\mathrm{t}_{\mathrm{o}}^{\prime}-\mathrm{t}_{\mathrm{r}}^{\prime}\right)\right] \\
& =\frac{\mathrm{w}}{\alpha}-\frac{\mathrm{x}}{(\alpha+\mathrm{y})}\left(\frac{1}{\alpha}-\frac{1}{\alpha+\mathrm{y}}\right)\left[\mathrm{e}^{-(\alpha+\mathrm{y})\left(\mathrm{t}_{\mathrm{r}}^{\prime}-\mathrm{t}_{\mathrm{o}}^{\prime}\right)}-1\right]-\frac{\mathrm{x}}{\alpha+\mathrm{y}}\left(\mathrm{t}_{\mathrm{o}}^{\prime}-\mathrm{t}_{\mathrm{r}}^{\prime}\right) \\
& =\frac{\mathrm{w}}{\alpha}-\frac{\mathrm{xy}}{\alpha(\alpha+\mathrm{y})^{2}}\left[\mathrm{e}^{-(\alpha+\mathrm{y})\left(\mathrm{t}_{\mathrm{r}}^{\prime}-\mathrm{t}_{\mathrm{o}}^{\prime}\right)}-1\right]-\frac{\mathrm{x}}{(\alpha+\mathrm{y})}\left(\mathrm{t}_{\mathrm{o}}^{\prime}-\mathrm{t}_{\mathrm{r}}^{\prime}\right)
\end{aligned}
$$

(4.32)

Also the present value of the inventory holding cost in RW and OW are respectively given by

$$
\begin{aligned}
& \operatorname{ch}_{2} \int_{t_{s}^{\prime}}^{t_{r}^{\prime}} e^{-r t} I_{r}(t) d t=c h_{2} \int_{t_{s}^{\prime}}^{t_{r}^{\prime}} \frac{x e^{-r t}}{\beta+y}\left(e^{-(\beta+y)\left(t-t_{r}^{\prime}\right)}-1\right) d t
\end{aligned}
$$

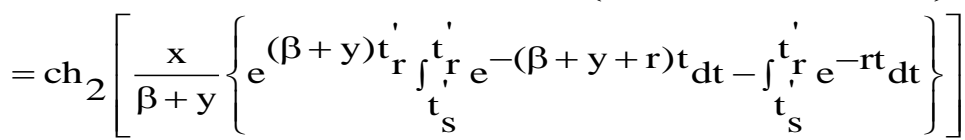

$$
\begin{aligned}
& =\frac{x_{2}}{\beta+y}\left[\frac{e^{(\beta+y) t_{r}^{\prime}}}{(\beta+y+r)}\left(e^{-(\beta+y+r) t_{s}^{\prime}}-e^{-(\beta+y+r) t_{r}^{\prime}}\right)-\frac{1}{r}\left(e^{-r^{\prime} s_{s}}-e^{-r t_{r}^{\prime}}\right)\right] \\
& =\frac{x_{2}}{\beta+y}\left[\frac{1}{(\beta+y+r)}\left(e^{-(\beta+y)\left(t_{s}^{\prime}-t_{r}^{\prime}\right)-r_{s}^{\prime}}-e^{-r_{r}^{\prime}}\right)-\frac{1}{r}\left(e^{-r_{t}^{\prime}}-e^{-r t_{r}^{\prime}}\right)\right] \\
& \operatorname{ch}_{2} \int_{t_{s}^{\prime}}^{t_{r}^{\prime}} e^{-r t_{r}} I_{r}(t) d t=\frac{x c h_{2}}{\beta+y}\left[\frac{e^{-r t_{s}^{\prime}}}{\beta+y+r}\left(e^{-(\beta+y)\left(t_{s}^{\prime}-t_{r}^{\prime}\right)}-e^{r\left(t_{s}^{\prime}-t_{r}^{\prime}\right)}\right)-\frac{e^{-r t_{s}^{\prime}}}{r}\left(1-e^{r\left(t_{s}^{\prime}-t_{r}^{\prime}\right)}\right)\right]
\end{aligned}
$$

and,

$$
\begin{aligned}
& \operatorname{ch}_{1} \int_{t_{s}^{\prime}}^{t^{\prime}} e^{-r t} I_{0}(t) d t=c h_{1}\left[\int_{t_{s}^{\prime}}^{t^{\prime}} r_{w} e^{\alpha t_{s}^{\prime}} e^{-(\alpha+r) t} d t\right. \\
& +\int_{t_{r}^{\prime}}^{t^{\prime}} \frac{x}{(\alpha+y)}\left(e^{(\alpha+y) t^{\prime}} e^{-(\alpha+y+r) t}-e^{-r t}\right) d t \\
& =\operatorname{ch}_{1}\left[\frac{w e^{\alpha t_{s}^{\prime}}}{(\alpha+r)}\left(e^{-(\alpha+r) t_{s}^{\prime}}-e^{-(\alpha+r) t_{r}^{\prime}}\right)+\right. \\
& \left.\frac{x}{(\alpha+y)}\left\{\frac{e^{(\alpha+y) t^{\prime}}}{(\alpha+y+r)}\left(e^{-(\alpha+y+r) t_{r}^{\prime}}-e^{-(\alpha+y+r) t_{0}^{\prime}}\right)-\frac{1}{r}\left(e^{-r_{r}^{\prime}} r^{-r t_{o}^{\prime}}\right)\right\}\right] \\
& \mathrm{ch}_{1} \int_{t_{\mathrm{s}}}^{\mathrm{t}^{\prime}}, \mathrm{o} \mathrm{e}^{-\mathrm{rt}} \mathrm{I}_{\mathrm{o}}(\mathrm{t}) \mathrm{dt}=\mathrm{ch}_{1}\left[\frac{\mathrm{we}}{(\alpha+\mathrm{r})}\left(1-\mathrm{e}^{-(\alpha+\mathrm{r})\left(\mathrm{t}_{\mathrm{r}}^{\prime}-\mathrm{t}_{\mathrm{s}}^{\prime}\right)}\right)+\right.
\end{aligned}
$$

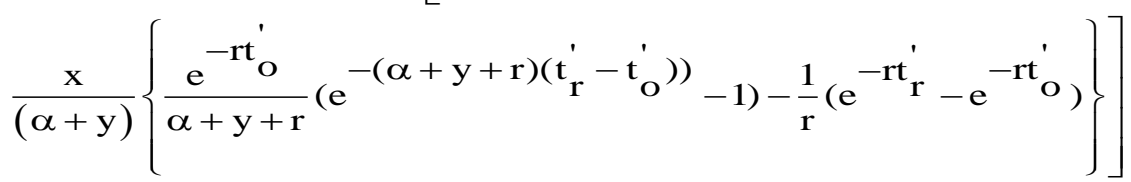

respectively and the present value of the backlogging cost and the opportunity cost due to lost sale are $c_{b} \int_{O}^{t^{\prime}} s^{-r t} \int_{O}^{t} \delta\left(t_{s}-u\right) x d u d t=\frac{c_{b}}{r} \int_{O}^{t^{\prime}} e^{-r}\left(e^{-r t}-e^{-r t^{\prime}} s\right) \delta\left(t_{s}^{\prime}-t\right) x d t$ 
And

$c_{1} e^{-r t^{\prime}} \int_{0}^{t^{\prime}} s\left\{1-\delta\left(t_{s}^{\prime}-t\right)\right\} x d t$

(4.36)

In addition, the present value of the cost for the deteriorated items is

$$
\begin{aligned}
& c_{p}\left[\beta \int_{t_{s}^{\prime}}^{t_{r}^{\prime}} e^{-r t} I_{r}(t) d t+\alpha \int_{t_{s}^{\prime}}^{t_{i o}^{\prime}} e^{-r t} I_{o}(t) d t\right] \\
& =c_{p}\left[\frac{\beta x}{\beta+y}\left\{\frac{e^{-r t_{s}^{\prime}}}{\beta+y+r}\left(e^{-(\beta+y)\left(t_{s}^{\prime}-t_{r}^{\prime}\right)}-e^{r\left(t_{s}^{\prime}-t_{r}^{\prime}\right)}\right)-\frac{e^{-r t_{s}^{\prime}}}{r}\left(1-e^{r\left(t_{s}^{\prime}-t_{r}^{\prime}\right)}\right)\right\}\right. \\
& +\frac{\alpha w e^{-r t_{s}^{\prime}}}{\alpha+r}\left(1-e^{-(\alpha+r)\left(t_{r}^{\prime}-t_{s}^{\prime}\right)}\right)+\frac{\alpha x}{\alpha+y}\left\{\frac{e^{-r t_{o}^{\prime}}}{\alpha+y+r}\left(e^{-(\alpha+y+r)\left(t_{r}^{\prime}-t_{o}^{\prime}\right)}-1\right)\right. \\
& \left.\left.-\frac{1}{r}\left(e^{-r t_{r}^{\prime}}-e^{-r t_{o}^{\prime}}\right)\right\}\right]
\end{aligned}
$$

Therefore, the present value of the total cost per unit time is given by

$$
\begin{aligned}
& \mathrm{Tc}_{2}=\frac{\mathrm{e}^{-\mathrm{rt}} \mathrm{s}}{\left(\mathrm{t}_{\mathrm{s}}{ }^{\prime}+\Delta_{3}+\Delta_{4}\right)}\left[\mathrm{c}_{\mathrm{o}}+\frac{\left(\mathrm{ch}_{2}+\beta \mathrm{c}_{\mathrm{p}}\right) \mathrm{x}}{\beta+\mathrm{y}}\left\{\frac{1}{\beta+\mathrm{y}+\mathrm{r}}\left(\mathrm{e}^{(\beta+\mathrm{y}) \Delta_{3}}-\mathrm{e}^{-\mathrm{r} \Delta_{3}}\right)-\frac{1}{\mathrm{r}}\left(1-\mathrm{e}^{-\mathrm{r} \Delta_{3}}\right)\right\}\right. \\
& +\frac{\left(\mathrm{ch}_{1}+\alpha \mathrm{c}_{\mathrm{p}}\right)}{\alpha+\mathrm{y}} \times \mathrm{e}^{-\mathrm{r}\left(\Delta_{3}+\Delta_{4}\right)}\left\{\frac{1}{\alpha+\mathrm{y}+\mathrm{r}}\left(\mathrm{e}^{(\alpha+\mathrm{y}+\mathrm{r}) \Delta_{4}}-1\right)-\frac{1}{\mathrm{r}}\left(\mathrm{e}^{\mathrm{r} \Delta_{4}}-1\right)\right\} \\
& +\frac{\left(\mathrm{ch}_{1}+\alpha \mathrm{c}_{\mathrm{p}}\right) \mathrm{w}}{\alpha+\mathrm{r}}\left(1-\mathrm{e}^{\left.-(\alpha+\mathrm{r}) \Delta_{3}\right)}+\mathrm{x} \int_{\mathrm{O}}^{\mathrm{t}^{\prime}}\left\{\frac{\mathrm{c}_{\mathrm{b}}}{\mathrm{r}}\left(\mathrm{e}^{\mathrm{r}\left(\mathrm{t}_{\mathrm{s}}^{\prime}-\mathrm{t}\right)}-1\right) \delta\left(\mathrm{t}_{\mathrm{s}}^{\prime}-\mathrm{t}\right)+\mathrm{c}_{1}\left(1-\delta\left(\mathrm{t}_{\mathrm{s}}^{\prime}-\mathrm{t}\right)\right)\right\} \mathrm{dt}\right.
\end{aligned}
$$

$$
\begin{aligned}
& \mathrm{w}=\frac{\mathrm{xe}^{-\alpha\left(\mathrm{t}_{\mathrm{s}}^{\prime}-\mathrm{t}_{\mathrm{r}}^{\prime}\right)}}{\alpha+\mathrm{y}}\left[\mathrm{e}^{-(\alpha+\mathrm{y})\left(\mathrm{t}_{\mathrm{r}}^{\prime}-\mathrm{t}_{\mathrm{o}}^{\prime}\right)}-1\right] \\
& \Delta_{3}=\mathrm{t}_{\mathrm{r}}^{\prime}-\mathrm{t}_{\mathrm{s}}^{\prime} \text { and } \Delta_{4}=\mathrm{t}_{\mathrm{o}}^{\prime}-\mathrm{t}_{\mathrm{r}}^{\prime}=\frac{1}{\alpha+\mathrm{y}} \ln \left(1+\frac{(\alpha+\mathrm{y}) \mathrm{we}^{\alpha\left(\mathrm{t}_{\mathrm{s}}^{\prime}-\mathrm{t}_{\mathrm{r}}^{\prime}\right)}}{\mathrm{x}}\right)
\end{aligned}
$$

\section{The Optimal Replenishment Policy:}

Now the optimal solution for both Models I and II will be established. For model I, we have $\frac{d \Delta_{1}}{d t_{r}}=\frac{d t_{o}}{d t_{r}}-1=\frac{-\alpha}{\alpha+y}\left[1-e^{-(\alpha+y) \Delta_{1}}\right]$

(4.39)

The necessary conditions for $\mathrm{TC}_{1}$ to be minimized are

$$
\begin{aligned}
& \frac{\partial T C_{1}}{\partial t r}=\left[\frac{\left(c h_{2}+\beta c_{p}\right) x}{\beta+y}\left\{\frac{(\beta+y) e^{(\beta+y) t_{r}+r e^{-r t} r}}{\beta+y+r}-e^{-r t_{r}}\right\}-\frac{\left(\mathrm{ch}_{1}+\alpha \mathrm{c}_{\mathrm{p}}\right) \mathrm{x}}{\alpha+\mathrm{y}} \times\right. \\
& \left\{e^{-r t_{r}}\left(1-e^{(\alpha+y) \Delta_{1}}\right)+\frac{(\alpha+y) e^{-r t}}{\alpha+y+r}\left[\left(1-e^{r \Delta_{1}}\right)-\frac{\alpha+r}{\alpha} e^{(\alpha+y+r) \Delta_{1}} \frac{d \Delta_{1}}{d t_{r}}+\frac{d \Delta_{1}}{d t_{r}}\right]\right\} \\
& +\mathrm{xe}^{-\mathrm{r}\left(\mathrm{t}_{\mathrm{r}}+\Delta_{1}+\Delta_{2}\right)}\left(1+\frac{\mathrm{d} \Delta_{1}}{\mathrm{dt}_{\mathrm{r}}}\right)\left\{\int _ { \mathrm { t } } ^ { \mathrm { t } } \left[\mathrm{c}_{\mathrm{o}} \delta\left(\mathrm{t}_{\mathrm{s}}-\mathrm{t}\right)-\mathrm{rc}_{1}\left(1-\delta\left(\mathrm{t}_{\mathrm{s}}-\mathrm{t}\right)\right)+\left(\frac{\mathrm{c}_{\mathrm{b}}}{\mathrm{r}}\left(\mathrm{e}^{\mathrm{r}\left(\mathrm{t}_{\mathrm{s}}-\mathrm{t}\right)}-1\right)-\mathrm{c}_{1}\right)\right.\right. \\
& \left.\left.\left.\delta^{\prime}\left(\mathrm{t}_{\mathrm{s}}-\mathrm{t}\right)\right] \mathrm{dt}-\left[\frac{\mathrm{c}_{\mathrm{b}}}{\mathrm{r}}\left(\mathrm{e}^{\mathrm{r} \Delta_{2}-1}\right) \delta\left(\Delta_{2}\right)+\mathrm{c}_{1}\left(1-\delta\left(\Delta_{2}\right)\right)\right]\right\}-\mathrm{TC}_{1}\left(1+\frac{\mathrm{d}_{1}}{\mathrm{dt}_{\mathrm{r}}}\right)\right] /\left(\mathrm{t}_{\mathrm{r}}+\Delta_{1}+\Delta_{2}\right)=0
\end{aligned}
$$


and

$$
\begin{aligned}
& \frac{\partial T C_{1}}{\partial \Delta_{2}}=x e^{-r\left(t_{r}+\Delta_{1}+\Delta_{2}\right)}\left\{\int _ { t _ { o } } ^ { t _ { s } } \left[c_{b} \delta\left(t_{s}-t\right)-r c_{1}\left(1-\delta\left(t_{s}-t\right)\right)+\left(\frac{c_{b}}{r}\left(e^{r\left(t_{s}-t\right)}-1\right)-c_{1}\right)\right.\right. \\
& \left.\left.\delta^{\prime}\left(\mathrm{t}_{\mathrm{s}}-\mathrm{t}\right)\right] \mathrm{dt}-\mathrm{TC}_{1}\right] /\left(\mathrm{t}_{\mathrm{r}}+\Delta_{1}+\Delta_{2}\right)=0
\end{aligned}
$$

From equations (4.40) and (4.41), the following can be easily obtained

$$
\begin{aligned}
& c_{S}\left(1-e^{-r \Delta_{2}}\right)=\frac{r(\alpha+y) e^{(\alpha+y+r) \Delta_{1}}}{\left(\alpha+y e^{(\alpha+y) \Delta_{1}}\right)}\left[\frac { ( c h _ { 2 } + \beta c _ { p } ) } { ( \beta + y + r ) } \left(e^{\left.(\beta+y+r) t_{r}-1\right)}\right.\right. \\
& -\frac{\left(c h_{1}+\alpha c_{p}\right)}{\alpha+y}\left(1-e^{(\alpha+y) \Delta_{1}}\right)-\frac{\left(c h_{1}+\alpha c_{p}\right)}{\alpha+y+r} \\
& \left\{\left(e^{\left.-r \Delta_{1}-1\right)-\frac{\alpha+r}{\alpha}} e^{(\alpha+y) \Delta_{1}} \frac{d \Delta_{1}}{d t_{r}}+e^{-r \Delta_{1}} \frac{d \Delta_{1}}{d t_{r}}\right\}\right]
\end{aligned}
$$

Thus $\Delta_{2}$ is also a function of $t_{r}$. Consequently, if $t_{r}$ is known, then $\Delta_{1}$ and $\Delta_{2}$ can be uniquely determined by using equations (4.18) and (4.42). The solution that satisfies (4.40) and (4.41) minimizes $\mathrm{TC}_{1}$ as follows $\frac{d t_{o}}{d t_{r}}=\frac{y}{\alpha+y}+\frac{\alpha}{\alpha+y} e^{-(\alpha+y) \Delta_{1}}>0$

(4.43)

Which implies that $\mathrm{t}_{0}$ is a strictly increasing function of $\mathrm{t}_{\mathrm{r} .}$. Next differentiating eq. (4.42) with respect to $t_{r}$, we obtain that $\frac{d t_{s}}{d t_{r}}>0$

Therefore, $t_{S}$ is also a strictly increasing function of $t_{r}$. Consequently if $t_{r}$ is given, equations (4.40) and (4.41) provides a unique solution for $t_{o}$ and $t_{S}$. Further we find that

$$
\frac{\partial^{2} T C_{1}}{\partial t_{r}{ }^{2}}=-\left(\frac{\partial^{2} T C_{1}}{\partial t_{r} \partial \Delta_{1}} \frac{d \Delta_{1}}{d t_{r}}+\frac{\partial^{2} T C_{1}}{\partial t_{r} \partial \Delta_{2}} \frac{d \Delta_{2}}{d t_{r}}\right)>0
$$

As a result, the solution minimizes $\mathrm{TC}_{1}$. Similarly, for model II, for given $\Delta_{3}$ the solution that satisfies the equations $\frac{\partial T C_{2}}{\partial t_{s}{ }^{\prime}}=\mathrm{o}$ and $\frac{\partial T C_{2}}{\partial \Delta_{3}}=\mathrm{o}$ minimizes $\mathrm{TC}_{2}$.

\section{Numerical Examples:}

$$
\begin{array}{rlllrl}
\text { Let } \mathrm{D} & =450, & \mathrm{c}_{0}=80, & \mathrm{c}_{\mathrm{h} 1}=1.5, & \mathrm{c}_{\mathrm{h} 2}=2.5, & \mathrm{c}_{\mathrm{S}}=0.2, \\
\mathrm{c}_{\mathrm{p}}=10, & \mathrm{w}=70, & \alpha=0.02, & \beta=0.04, & \text { and } & \mathrm{r}=0.06,
\end{array}
$$

The computational results for the two models are shown below:

\begin{tabular}{|c|c|}
\hline Model I & Model II \\
\hline $\mathrm{t}_{\mathrm{r}}=0.0491$ & $\mathrm{t}_{\mathrm{s}}=1.2736$ \\
\hline$\Delta_{1}=0.2177$ & $\Delta_{3}=0.0373$ \\
\hline$\Delta_{2}=1.1374$ & $\Delta_{4}=0.8450$ \\
\hline $\mathrm{TC}_{1}=223.5732$ & $\mathrm{TC}_{2}=216.6314$ \\
\hline
\end{tabular}

Sensitivity analysis of parameters on $\mathrm{TC}_{1}-\mathrm{TC}_{2}$

\begin{tabular}{|c|c|c|c|}
\hline Parameter & Percentage of change $\%$ & $\mathrm{TC}_{1}-\mathrm{TC}_{2}$ & S. Rate $(\%)$ \\
\hline \multirow{2}{*}{$\mathrm{R}$} & +20 & 8.672 & 0.89 \\
\cline { 2 - 4 } & -20 & 3.531 & 1.06 \\
\hline \multirow{2}{*}{$\mathrm{C}_{\mathrm{o}}$} & +20 & 4.732 & 3.73 \\
\cline { 2 - 4 } & -20 & 4.317 & 2,53 \\
\hline \multirow{2}{*}{$\mathrm{C}_{\mathrm{s}}$} & +20 & 3.139 & 2.53 \\
\cline { 2 - 4 } & -20 & 2.984 & 2.95 \\
\hline $\mathrm{C}_{\mathrm{h} 1}$ & +20 & 4.0132 & 2.45 \\
\cline { 2 - 4 } & -20 & 3.891 & 1.48 \\
\hline $\mathrm{C}_{\mathrm{h} 2}$ & +20 & 3.884 & 1.85 \\
\hline
\end{tabular}




\begin{tabular}{|c|c|c|c|}
\hline & -20 & 4.205 & 1.68 \\
\hline \multirow{2}{*}{$\mathrm{C}_{\mathrm{p}}$} & +20 & 4.349 & 1.52 \\
\cline { 2 - 4 } & -20 & 3.993 & 1.96 \\
\hline \multirow{2}{*}{$\mathrm{A}$} & +20 & 4.0825 & 1.75 \\
& -20 & 3.963 & 1.85 \\
\hline \multirow{2}{*}{$\mathrm{B}$} & +20 & 4.361 & 1.43 \\
\cline { 2 - 4 } & -20 & 3.782 & 1.78 \\
\hline
\end{tabular}

Note that "S Rate" in above table stands for the saving rate in the total relevant cost per unit time, which is given by

S Rate $=\left(\mathrm{TC}_{1}-\mathrm{TC}_{2}\right) / \mathrm{TC}_{2}$

\section{Observations:}

1. It is clear from above table, for $r>0$, the total cost per unit time for model II is less expensive to operate than that for model I

2. If $r=0$, then the total cost per unit time for both models are same.

\section{Conclusion:}

In this chapter, an alternative model for determining the optimal replenishment cycle for the twowarehouse inventory problem under inflation, in which the inventory deteriorates at a constant rate over time and shortages are allowed. We conclude that the proposed model is less expensive to operate than the traditional one if the inflation rate is greater than zero. However, if the inflation rate is zero, then the total relevant cost per unit time for both models is same. The two-warehouse model can be applied to many practical situations. At present, due to introduction of open market policy, the business competition becomes very high to occupy much more profit in the sales market. For this reason, in order to attract more customer a departmental store is forced to provide the customers a better purchasing-environment such as well decorated show-room with modern light and electronic arrangements and enough free space for choosing items. Again, due to the expending of market situation, there is a crisis of space in the market places especially in the super marker, corporation market etc. As a result, the management of departmental store is bounded to hire a separate warehouse on rental basis at a distance place for storing of excess items. Hence, from the economical point of view, the two-storage system is more profitable than the single storage system.

\section{References:}

[1] Benkherouf, L. (1997): A deterministic order level inventory model for deteriorating items with two storage facilities. I.J.P.E., 48 (2), $167-175$.

[2] Bhunia, A.K. and Maiti, M. (1998): A two-warehouse inventory model for deteriorating items with a linear trend in demand and shortages. J.O.R.S., 49 (3), 287-292.

[3] Goswami, A. and Chaudhuri, K.S. (1992): An Economic order quantity model for items with two levels of storage for a linear trend in demand. J.O.R.S., 43, 157-167.

[4] Hsieh, T.P., Dye, C.Y. and Ouyang, L.Y. (2008): Determining optimal lot size for a two-warehouse system with deterioration and shortages using net present value. E.J.O.R., 191 (1), 180-190.

[5] Lee, C.C. (2006): Two-warehouse inventory model with deterioration under FIFO dispatching policy. E.J.O.R., 174 (2), 861-873

[6] Murdeshwar, T.M. and Sathe, Y.S. (1985): Some aspects of lot size models with two levels of storage. Opsearch, 22, 255-262.

[7] Pakkala, T.P.M., Achary, K.K. (1992): A deterministic inventory model for deteriorating items with two warehouses and finite replenishment rate. E.J.O.R., 57, 71-76.

[8] Pakkala, T.P.M., Achary, K.K. (1994): Two level storage inventory model for deteriorating items with bulk release rule. Opsearch, 31, 215-227.

[9] Sarma, K.V.S. (1983): A deterministic inventory model with two level of storage and an optimum release rule. Opsearch, 20(3), 175180

[10] Sarma, K.V.S. (1987): A deterministic order level inventory model for deteriorating items with two storage facilities. E.J.O.R., 29, 70-73.

[11] Yang, H.L. (2004): Two warehouse inventory models for deteriorating items with shortages under inflation, E.J.O.R., 157, $344-356$.

[12] Zhou, Y.W. and Yang, S.L. (2005): A two-warehouse inventory model for items with stock-level-dependent demand rate. I.J.P.E., 95 (2), 215-228. 